\title{
Using Absolutist Word Frequency from Online Searches to Measure Population Mental Health Dynamics
}

\author{
Jais Adam-Troian', Thomas Arciszewski², Eric Bonetto ${ }^{2}$
}

\section{Highlights}

Absolutist word use is a semantic marker for depression.

Absolutist word frequency from online searches is linked with population rates of depression in the UK, $r=.68,95 \% \mathrm{CI}[.34, .86]$, and in France, $\beta=.23,95 \% \mathrm{CI}[.09, .37]$.

Absolutist word frequency from online searches by 3.2\%, 95\%CI[2.1,4.2] in France and $3.7 \%, 95 \% \mathrm{CI}[2.9,4.4]$ in the UK in 2020 compared to 2019.

Absolutist word frequency from online searches co-varies with the pandemic waves, and is related to COVID-19 new deaths in both countries $\beta=.14,95 \% \mathrm{CI}[.14, .21]$.

Absolutist word frequency from online searches seems to be a valid, cost-effective "thermometer" for public mental health - at least in Western Europe.

\begin{abstract}
The assessment of population mental health relies on survey data from representative samples, which come with considerable costs. Drawing on research which established that absolutist words (e.g. never) are semantic markers for depression, we propose a new measure of population mental health based on the frequency of absolutist words in online search data (Absolute Thinking Index; ATI). Our aims were to first validate the ATI, and to use it to model public mental health dynamics in France and the UK during the current COVID-19 pandemic. To do so, we extracted time series for a validated dictionary of 19 absolutist words, from which the ATI was computed (weekly averages, 2019-2020, $n=208$ ). We then tested the relationship between ATI and longitudinal survey data of population mental health in the UK and France. ATI was linked with survey depression scores in the UK, $r=.68,95 \% \mathrm{CI}[.34, .86], \beta=.23$, 95\%CI[.09,.37] in France and displayed similar trends. We finally assessed the pandemic's impact on ATI using Bayesian structural time-series models. These revealed that the pandemic increased ATI by 3.2\%, 95\%CI[2.1,4.2] in France and 3.7\%, 95\%CI[2.9,4.4] in the UK. Mixedeffects models showed that ATI was related to COVID-19 new deaths in both countries $\beta=.14$, $95 \%$ CI[.14,.21]. Our results demonstrate the validity of the ATI as a measure of population mental health (depression) in France and the UK. We propose that researchers use it as costeffective public mental health "thermometer" for applied and research purposes.
\end{abstract}

\section{Funding}

None.

\footnotetext{
${ }^{1}$ American University of Sharjah

${ }^{2}$ Aix-Marseille University
} 


\section{INTRODUCTION}

The ongoing COVID-19 pandemic context has generated global consequences for public mental health ${ }^{1}$. Growing evidence from longitudinal studies suggests worldwide increases in PTSD, depression and anxiety disorders in 2020 compared to previous years ${ }^{2-5}$. Research suggests that these consequences result from the combination of lockdown measures (i.e. social isolation $)^{6-7}$, economic downturn ${ }^{8}$ and fear of the pandemic itself ${ }^{9-10}$. In the US for instance, depression levels increased significantly during the pandemic (April 2020), but even more so among young adults (age 18-34) ${ }^{11}$. Understanding and monitoring the extent to which the pandemic affects public mental health is therefore critical to prevent indirect increases in suicide and substance abuse-related deaths during pandemics ${ }^{12-13}$.

Proper monitoring of public mental health would allow to quantify the 'side-effects' of policies aimed to slow down the spread of COVID-19 ${ }^{14}$, enabling to factor those in costbenefit analyses to assist decision making. However, current techniques to monitor population mental health rely heavily on self-report data ${ }^{2-10}$. This is problematic because longitudinal surveys conducted on representative samples of any population are costly to implement, and necessarily involve complex methodological challenges (such as dealing with attrition ${ }^{15}$ ). More importantly maybe, longitudinal survey studies usually provide a handful of time points and are often conducted in reaction to events, which prevent the use of more powerful analytical techniques for causal inference ${ }^{16-17}$.

To tackle these issues, we propose a novel behavioral measure of public mental health that allows for cheap, continuous probing of population mental health levels. To do so, we draw on cognitive theories of depression, which established that absolutist thinking is associated with anxiety, depression and suicidal ideation ${ }^{18-21}$. Accordingly, studies show that absolutist words (e.g. completely, totally, all) are more prevalent among depressed, suicidal patients and in writings of suicidal individuals ${ }^{18-21}$. The frequency of absolutist word use in speech is 
therefore a reliable linguistic marker for depression and related disorders. Recent investigations have leveraged this marker using search engine data (e.g. Google Trends ${ }^{22}$ ) to show how an index of absolutist word frequency in online searches (Absolute Thinking Index, ATI) can predict state-level suicide rates in the $\mathrm{US}^{23}$.

The ATI approach is partly in line with previous research using online search behavior data to attempt to measure population mental health ${ }^{24,25}$ by looking at frequency of search words related to mental health (e.g. OCD, anxiety, suicide). The novelty of this method is that it does not rely on the meaning of searched words, but on their psychological function ${ }^{26}$. Indeed, the focus on meaning rests on strong untested assumptions, for instance that searching for mental health symptoms is a reliable marker for mental health diagnosis, and generates sensitivity to sentence wording (e.g. inclusion of question mark or not). This has led researchers to question the validity of measures based on online search data ${ }^{27-28}$. In comparison, the ATI's only assumption is that the way individuals typewrite is influenced by their mood, an uncontroversial assumption which has empirically been verified recently ${ }^{29}$.

In fact, the ATI approach proposes that frequency of absolutist word "slips" in online searches - regardless of the searched topic itself - should be a reliable proxy for population mental health (and depression in particular). Given that absolutist word use indicates higher levels of depression, the likelihood of absolutist word "slips" in search requests should increase with the level of individual depressive symptoms (e.g. typing "is the airport totally shut down?" instead of "is the airport shut down?"). Drawing on the ATI method, the present study has therefore a double aim. First, we propose to validate the ATI for the first time - by leveraging survey data from longitudinal studies of mental health during the COVID-19 pandemic in the UK and in France. Second, we use the ATI to model the population mental health dynamic during the pandemic and to quantify its impact.

\section{METHODS}




\section{Ethical statement}

The study was conducted in accordance with the 2016 APA Ethical Principles of Psychologists and Code of Conduct. Ethic approval requirement was waived due to the nature of our data (publicly accessible anonymous archival data on aggregates). The data underlying our findings is openly accessible at

\section{https://osf.io/2yh65/?view_only=e750deb7ac0a46358cab870e3fb1d260}

\section{Online search data}

To compute the ATI, we used search volume data from Google Trends. In short, Google Trends provides researchers with the weekly frequency at which a specific search term is typed, compared to all different search terms across specified languages and geographical $\operatorname{areas}^{22}$. These data are publicly available and can be easily retrieved. When extracting data on a specific word in Google Trends it is possible to retrieve its prevalence among all search entries in a given population. For example, data extracted for the word "completely" counts the relative frequency at which this word was typed in every request in the specified geographical area during a given time period.

\section{Data extraction}

The criteria for country selection was 1) survey data availability 2) over a sufficiently long period of time in countries 3) displaying similar pandemic dynamics and government responses for comparability. We therefore focused our efforts on the UK and France, which met all criteria. For each time period in France and in the UK, we proceeded similarly by collecting search volume time series for word searches regarding the 19 terms from the absolutist language dictionary ${ }^{18,23}$ (“absolutely," "all," "always”, “complete," “completely," “constant," “constantly," “definitely," “entire," "ever," "every," "everyone," “everything," “full," “must," “never," “nothing," "totally," “whole”). 
Whenever data was too few and the output indicated an estimate " $<1$ ", we rounded it down to 0 to allow for quantitative analysis (those occurrences were overall rare though, $<1 \%$ of total data). In French, the words for "completely" (“complètement') and "ever" (“déjà") are written with accents, so we also extracted them without accents because individuals sometimes omit them to type faster. The extracted data thus consisted in 19 time series ( 21 for France) from which we computed a single sum score variable (ATI) after checking for reliability.

For the validation analysis in the UK, we computed the ATI in England from march $23^{\text {rd }}$ to August $9^{\text {th }}$ of $2020(\alpha=.76)$ to match longitudinal survey data of anxiety and depression collected from the post-lockdown UCL COVID-19 Social Study ${ }^{30}(n=20)$. We also computed a previously used search data-based measure of common mental health symptoms ${ }^{25}$ to examine how it would perform (averaging the following words: "anxiety," "depression," “OCD”, "hopeless," "angry," "afraid," “apathy," “worthless," "worried,” "restless,” “irritable," "tense," “scattered," "tired," “avoiding," "procrastinate," “insomnia," "suicidal," and "suicide").

Similarly for the validation analysis in France, we computed the ATI (using regional-level data this time; $\alpha=.74$ ) to match data from the COVIPREV longitudinal survey (see https://www.santepubliquefrance.fr/etudes-et-enquetes/coviprev-une-enquete-pour-suivre-1evolution-des-comportements-et-de-la-sante-mentale-pendant-1-epidemie-de-covid-19) from the week of march $23^{\text {rd }}$ to that of October $19^{\text {th }}$ of 2020 (16 weekly waves, 12 regions, $n=$ 192). Finally, we computed the ATI for the whole of 2019 and 2020 in both countries for the pandemic impact analysis $(\alpha=.86 ; \mathrm{n}=208)$.

\section{RESULTS}

\section{Analysis 1 - UK Validation}


Results from Pearson correlation analyses indicated that ATI was linked with survey anxiety, $r(20)=.68,95 \% \mathrm{CI}[.34, .86], p<.001$, as well as depression measures $r(20)=.83$, 95\% CI[.60,.92], $p<.001$ (see figure 1). ATI was also correlated with average search frequency for mental health-related terms $r(20)=.46,95 \% \mathrm{CI}[.03, .75], p=.04$, although this last measure was not substantially related to either survey anxiety $r(20)=.13,95 \% \mathrm{CI}[-.33, .54]$, $p=.59$ or depression measures, $r(20)=.34,95 \% \mathrm{CI}[-.13, .68], p=.15$.

Yet, because our data are essentially time series, we needed to rule out non-independence of observations as a potential source of bias. To do so, we regressed all our measures on time and extracted the remaining residuals of these analyses. All variables exhibited linear decay, $\beta$ $=-.82,95 \% \mathrm{CI}[-1.10,-.54], p<.001$ for ATI, $\beta=-.94,95 \% \mathrm{CI}[-1.11,-.78], p<.001$ for anxiety and $\beta=-.71,95 \% \mathrm{CI}[-1.06,-.36], p<.001$, for depression - at the exception of average frequency of mental health searches $\beta=.23,95 \% \mathrm{CI}[-.71, .25], p=.33$. We then re-ran Pearson correlations using the residuals. Although the link between ATI and depression held, $r(20)=$ $.61, p=.006$, the relationship with anxiety reversed, $r(20)=-.50, p=.03$. suggesting a potential artefactual origin (i.e. from data non-independence).

This first validation analysis therefore provided evidence for predictive, concurrent and construct validity of the ATI. Not only this measure was strongly linked with survey measure of depression and, but it also displayed a similar dynamic over time than what was found in the UCL COVID-19 Social Study's results ${ }^{30}$. However, the sample size was small and we still needed to establish cross-cultural validity of the ATI before using it as a proxy for population mental health in France. We therefore performed a second validation. 


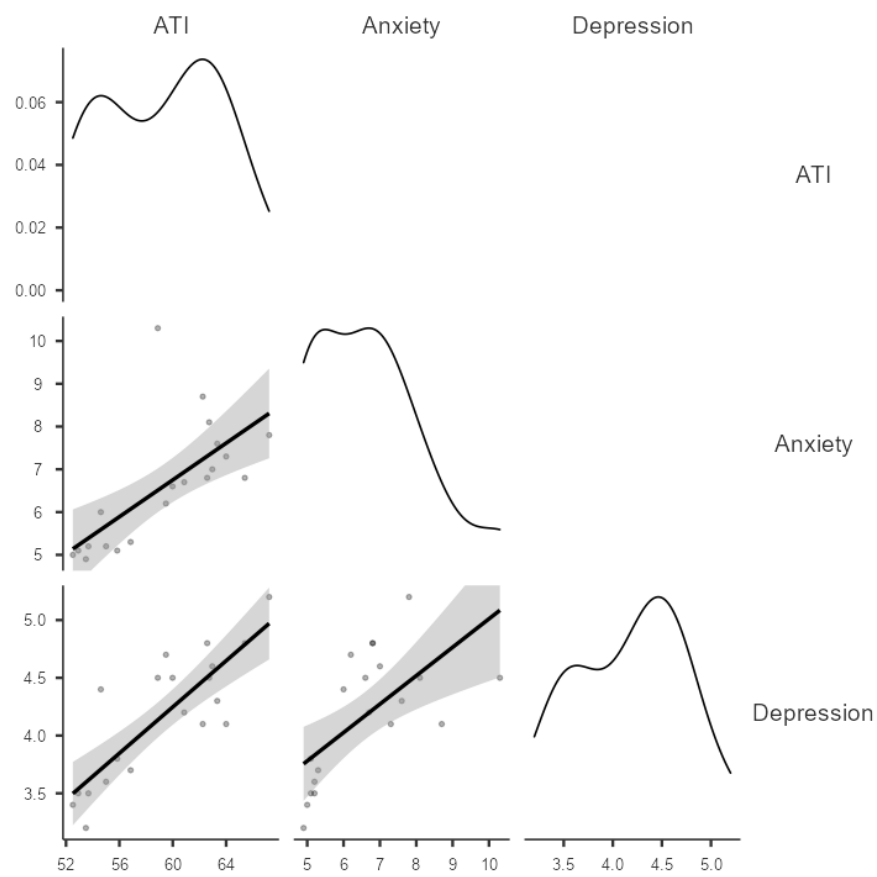

Figure 1. Correlation plots for ATI, survey Anxiety and Depression measures. Grey areas indicate SEs and distribution shapes densities.

\section{Analysis 2 - France Validation}

In France, the larger number of observations and the data structure allowed to proceed with finer grained analyses. The only difference was that depression and anxiety measures in that sample reflect provisional diagnoses for disorders, not scores (i.e. percentage of individual above scale cutoff scores). To account for clustering within regions $(\mathrm{n}=12)$ and to directly incorporate time-related trends (survey waves, $\mathrm{n}=16$ ), mixed-effects models were computed for each survey measure allowing both slopes and intercepts to vary according to the following specifications:

(1) depression $=1+A T I+$ wave $+(1+A T I+$ wave $\mid$ region $)$

(2) anxiety $=1+A T I+$ wave $+(1+A T I+$ wave $\mid$ region $)$

Results replicated what we found in the UK, with $1 \%$ ATI increase associated with $+.23 \%$ depression diagnoses $95 \% \mathrm{CI}[.09, .37], t(22)=3.30, p=.003$, while it was not robustly linked 
with anxiety $\beta=.20,95 \% \mathrm{CI}[-.01, .38], p=.076$ (full model tables are available at https://osf.io/2yh65/?view_only=e750deb7ac0a46358cab870e3fb1d260).

Interestingly, all three variables seemed to display a polynomial dynamic, with an initial decline and stabilization until week 10 (June $8^{\text {th }}$ ) and a progressive increase again (see figure 2). This was confirmed by further mixed-modelling according to the following specifications:

(3) depression $=1+$ wave + wave $^{2}+$ wave $^{3}(1 \mid$ region $)$

(4) anxiety $=1+$ wave + wave $^{2}+$ wave $^{3}(1 \mid$ region $)$

(5) $A T I=1+$ wave + wave $^{2}+$ wave $^{3}(1 \mid$ region $)$

Although anxiety rates displayed a second-order (quadratic) trend, $\beta=.22,95 \% \mathrm{CI}[.07, .35], p$ $=.005$ (cubic parameter $\mathrm{p}>.10$, see full model on the OSF project page), both ATI and depression rates displayed similar cubic behavior with respectively $\beta=.01,95 \%$ CI[.006,.013], $p<.001$ and $\beta=.02,95 \% \mathrm{CI}[.01, .03], p<.001$. This provided further evidence for the specificity of ATI - which mimicked depression dynamics, and the polynomial trend observed was indicative of something ongoing around the same time the $2^{\text {nd }}$ wave of the pandemic started in France. Having established the relevance of using ATI as a proxy for mental health (and more specifically depression) in both the UK and France, we proceeded to our main analysis of the pandemic's effect on population mental health. 


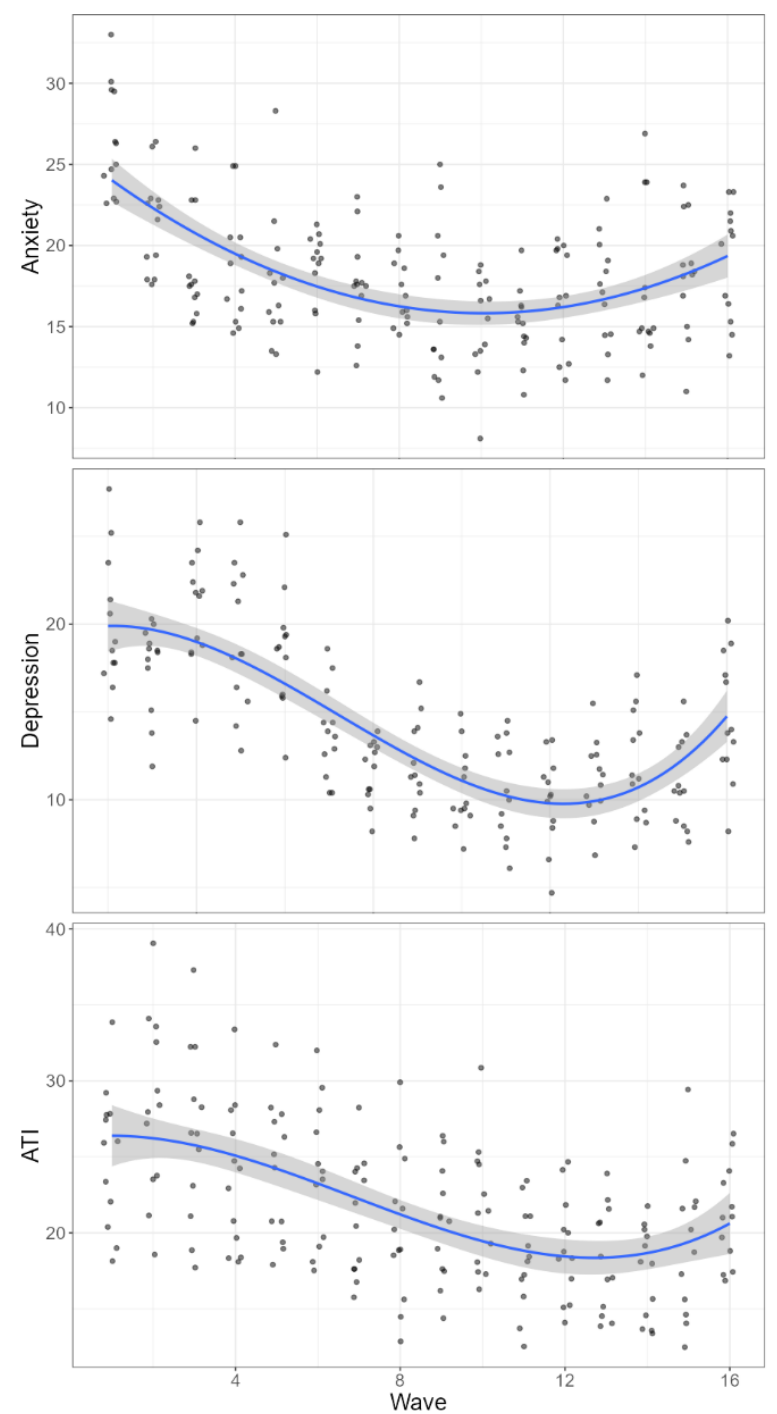

Figure 2. Chronogram for ATI, Anxiety and Depression measures across regions and over the weeks from March $23^{\text {rd }}$ (wave 1) to October $19^{\text {th }}$ (wave 16). Blue fitted lines represent second-order polynomial for Anxiety and third-order for ATI and depression. Grey areas represent SEs.

\section{Analysis 3 - Joint Pandemic Impact Analysis}

For this third analysis, we combined weekly ATI data from 2019 and $2020(n=104)$ in both France and the UK (total $n=208$ ) As can be seen in figure 3, ATI dynamics are quite similar across countries, and most likely reflect a strong impact of the COVID-19 pandemic, at least during the first wave. 


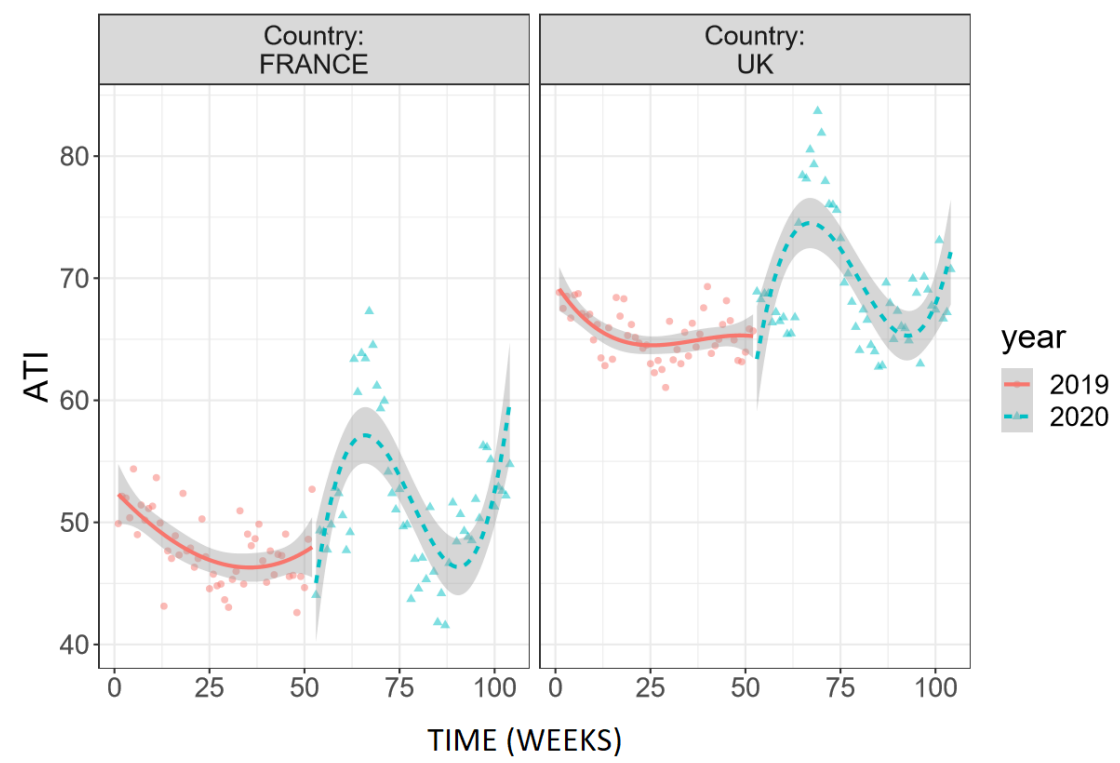

Figure 3. Chronogram for ATI, weekly measures across France and the UK and over the weeks from January $1^{\text {st }}$ of 2020 (1) to December $30^{\text {th }}$ of 2020 (104). Fitted lines represent polynomial for ATI and grey areas represent SEs.

We then set out to test this hypothesis using Bayesian structural time-series models ${ }^{30}$. In short, these models allow to train Markov chain Monte Carlo algorithm to quasi-experimentally generate the estimate of an exogeneous event's impact on a time series of interest relative to a time-series hypothesized to be unaffected by the said event. To do so, we extracted and averaged a series of 19 randomly generated words from Google Trends (using https://randomwordgenerator.com/) matched in English and French so as not to contain words with accents ( "position," “money," "seed”, "participate," "node," "asylum," “dominate,"

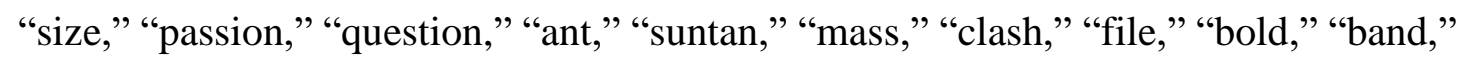
“addition," and "attachment").

After specifying the pre and pandemic periods based on reports of the first cases to appear in each country, (week 56 in France and 57 in the UK), we ran the Bayesian models (syntax, full tables and data available on the project OSF page). Our analyses indicate that, in line with the visual effect on figure 3, the pandemic did increase ATI by an absolute average 3.2\%, 95\% CI[2.1,4.2], $p=.001$ in France and by an average 3.7\%, 95\%CI[2.9,4.4], $p=.001$ in the UK (see figure 4). 


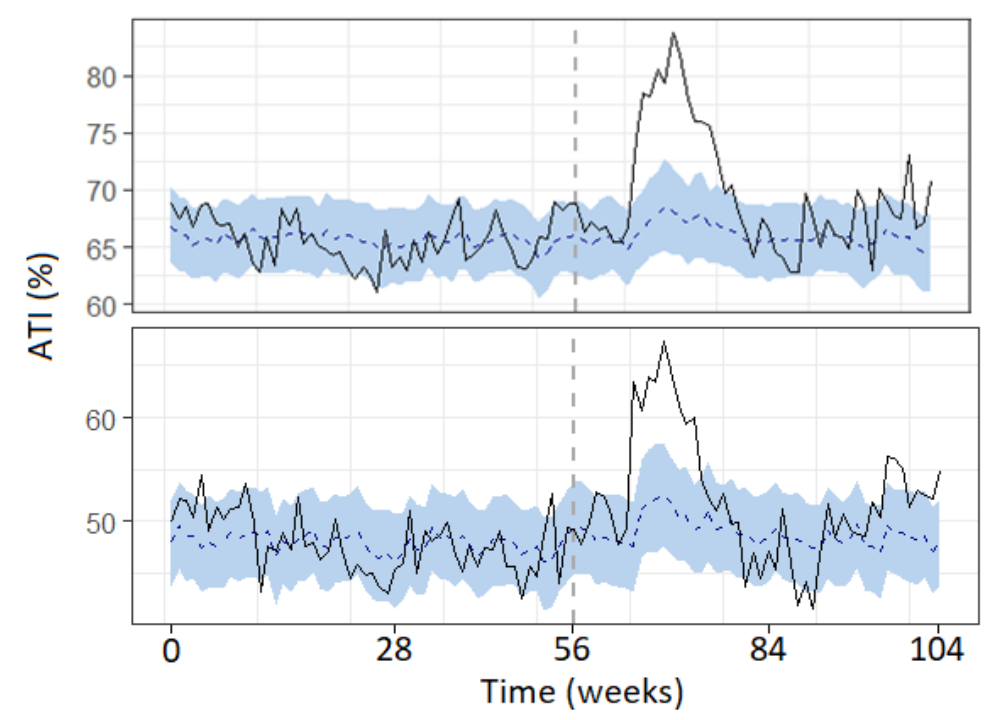

Figure 4. Chronogram of ATI weekly measures (dark line) relative to the synthetic predicted control time series (blue line). Measures are taken across France (below) and the UK (above) over the weeks from January $1^{\text {st }}$ of 2020 (1) to December $30^{\text {th }}$ of 2020 (104). Fitted lines represent polynomial for ATI and blue areas represent SEs.

Overall, we had strong evidence that the ATI was impacted by the pandemic, but we wished to further investigate this phenomenon. We decided to focus a last analysis on data during the pandemic exclusively, factoring in both new weekly cases and deaths per million inhabitants from the COVID-19 Data Repository by the Center for Systems Science and Engineering (CSSE) at Johns Hopkins University (available at https://github.com/CSSEGISandData/COVID-19) in France and the UK ( $\mathrm{n}=96)$. A final mixed-effects model was therefore computed according to the following (full model available on the OSF project page):

(6) $A T I=1+$ cases + deaths + weeks $(1 \mid$ country $)$

Despite a general tendency to go back to baseline over the weeks, $\beta=-.18,95 \% \mathrm{CI}[-.25,-.10]$, $p<.001$, ATI increased significantly as a function of COVID-19 deaths $\beta=.14$, $95 \% \mathrm{CI}[.14, .21], p<.001$, but not cases $\beta=-.001,95 \% \mathrm{CI}[-.001, .001], p=.40$ (see figure 5) 

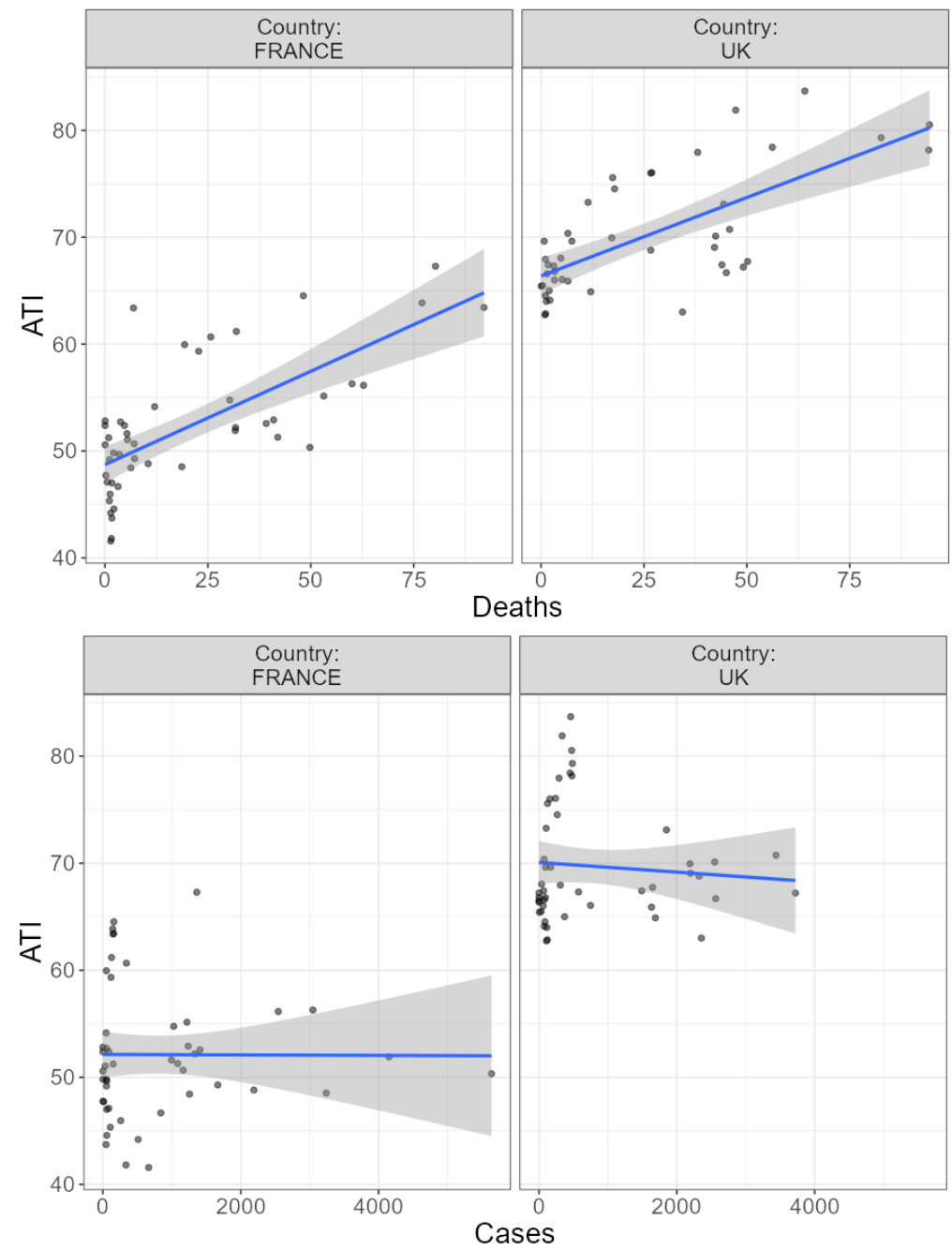

Figure 5. Scatterplot of ATI weekly measures as a function of COVID-19 deaths (above) and cases (below) in France (below) and the UK (above). Fitted blue lines represent linear trends for ATI and grey areas represent SEs.

\section{DISCUSSION}

This study aimed to test the validity of a new behavioral measure of mental health based on psycholinguistic and clinical psychology theory. Across analyses, we verified that ATI was robustly associated with depression measures, as theoretically expected, with different survey operationalization, in two languages and cultural settings. Critically, ATI displayed a decrease after the first lockdown in the UK, similar to survey measures of depression (Analysis 1). In addition, ATI had a third-order polynomic behavior over time in France, just as depression measures among the population but unlike anxiety (Analysis 2). This provided further evidence for discriminant validity of the ATI. 
Our impact analyses revealed that the ATI reacts just as population mental health issues was found to react during the pandemic, with a steep increase in the first wave and a decline as individuals adjust, to degrade once more with the second incoming wave (Analysis 3). The differences in ATI in 2020 compared to 2019 are also in line with estimates from various survey studies in the US and the $\mathrm{UK}^{2,9,10}$. In addition, the main driver of ATI increases seems to be COVID-19 deaths in the population, in line with what could be expected in terms of population-level depression increases as individuals lose close relatives. On the other hand, more substantial work needs to be conducted to disentangle conjoint economic (e.g. unemployment), policy (e.g. lockdown) and psychosocial (e.g. emotion contagion) effects of the pandemic on population mental health.

Overall, we believe that the ATI could be used as a potent multipurpose cost-effective monitor of population mental health. As discussed in earlier studies ${ }^{22}$, such a tool could be used to assess not only pandemic mental health consequences, but also the effect of public mental health policies, like suicide prevention campaigns. Retrospective analysis on local policies or targeted geographical areas may help discover community resilience factors of interest. Unusual ATI behavior in areas of interest may help diagnose at-risk communities and assist allocation of economic resources dedicated to improve population mental health outcomes. Sudden changes in ATI could also help discover emergent pandemics (e.g. substance abuse) before the effects are seen on hospital admissions for overdose. Finally, discrepancies between ATI and survey data may also be used to highlight methodological issues and mis-estimates of population mental health through traditional survey means.

Although more work needs to be conducted to guarantee the external validity of ATI as a measure of population mental health in other settings and languages, we believe to have established strong evidence in favor of considering the ATI for research and applied purposes in public mental health in Western European countries. 
Declaration of Interests: We declare no conflict of interests.

\section{REFRENCES}

1 - Torales, J., O’Higgins, M., Castaldelli-Maia, J. M., \& Ventriglio, A. (2020). The outbreak of COVID-19 coronavirus and its impact on global mental health. International Journal of Social Psychiatry, 66(4), 317-320.

2-Pierce, M., Hope, H., Ford, T., Hatch, S., Hotopf, M., John, A., ... \& Abel, K. M. (2020). Mental health before and during the COVID-19 pandemic: a longitudinal probability sample survey of the UK population. The Lancet Psychiatry, 7(10), 883-892.

3- Canet-Juric, Lorena, et al. "A longitudinal study on the emotional impact cause by the COVID-19 pandemic quarantine on general population." Frontiers in Psychology 11 (2020): 2431.

4- Xiong, Jiaqi, et al. "Impact of COVID-19 pandemic on mental health in the general population: A systematic review." Journal of affective disorders (2020).

5- Adams-Prassl, Abi, et al. "The impact of the coronavirus Lockdown on mental health: evidence from the US." (2020).

6- Galea S, Merchant RM, Lurie N. The mental health consequences of COVID-19 and physical distancing: the need for prevention and early intervention.JAMA Intern Med. 2020;180(6):817- 818. doi:10.1001/jamainternmed.2020.1562

7- Witteveen, Dirk, and Eva Velthorst. "Economic hardship and mental health complaints during COVID-19." Proceedings of the National Academy of Sciences 117.44 (2020): 27277 27284.

8- Rahman, M. A., Hoque, N., Alif, S. M., Salehin, M., Islam, S. M. S., Banik, B., ... \& Cross, W. (2020). Factors associated with psychological distress, fear and coping strategies during the COVID-19 pandemic in Australia. Globalization and Health, 16(1), 1-15.

9- French, Michael T., Karoline Mortensen, and Andrew R. Timming. "Psychological Distress and Coronavirus Fears During the Initial Phase of the COVID-19 Pandemic in the United States." The Journal of Mental Health Policy and Economics 23.3 (2020): 93-100.

10 - Daly, M., Sutin, A. R., \& Robinson, E. (2021). Depression reported by US adults in 2017-2018 and March and April 2020. Journal of Affective Disorders, 278, 131-135.

11 - Gunnell D, Appleby L, Arensman E, et al; COVID-19 Suicide Prevention Research Collaboration. Suicide risk and prevention during the COVID-19 pandemic. Lancet Psychiatry. 2020;7 (6):468-471. doi:10.1016/S2215-0366(20)30171-1

12 - Holland, Kristin M., et al. "Trends in US emergency department visits for mental health, overdose, and violence outcomes before and during the COVID-19 pandemic." JAMA psychiatry (2021).

13 - Haug, Nils, et al. "Ranking the effectiveness of worldwide COVID-19 government interventions." Nature human behaviour 4.12 (2020): 1303-1312. 
14 - Twisk, Jos, and Wieke de Vente. "Attrition in longitudinal studies: how to deal with missing data." Journal of clinical epidemiology 55.4 (2002): 329-337.

15 - Sims, C. A. (1980). Macroeconomics and reality. Econometrica: journal of the Econometric Society, 1-48.

16 - Toda, H. Y., \& Yamamoto, T. (1995). Statistical inference in vector autoregressions with possibly integrated processes. Journal of econometrics, 66(1-2), 225-250.

17 - Al-Mosaiwi, Mohammed, and Tom Johnstone. "In an absolute state: Elevated use of absolutist words is a marker specific to anxiety, depression, and suicidal ideation." Clinical Psychological Science 6.4 (2018): 529-542.

18 - Neuringer, Charles. "Rigid thinking in suicidal individuals." Journal of consulting psychology 28.1 (1964): 54.

19 - Wedding, Danny. "Cognitive distortions in the poetry of Anne Sexton." Suicide and LifeThreatening Behavior 30.2 (2000): 140-144.

20 - Ellis, Thomas E., and Billy Rutherford. "Cognition and suicide: Two decades of progress." International Journal of Cognitive Therapy 1.1 (2008): 47-68.

21 - Google. (2017). Google Trends. https://trends.google.com/trends

22 - Adam-Troian, Jais, and Thomas Arciszewski. "Absolutist Words From Search Volume Data Predict State-Level Suicide Rates in the United States." Clinical Psychological Science 8.4 (2020): 788-793.

23 - Knipe, Duleeka, et al. "Mapping population mental health concerns related to COVID-19 and the consequences of physical distancing: a Google trends analysis." Wellcome Open Research 5 (2020).

24- Jacobson, Nicholas C., et al. "Flattening the mental health curve: COVID-19 stay-at-home orders are associated with alterations in mental health search behavior in the United States." JMIR mental health 7.6 (2020): e19347.

25- Jackson, Joshua, et al. "From text to thought: How analyzing language can advance psychological science." (2020).

26 - Fond, Guillaume, et al. "Google Trends®: Ready for real-time suicide prevention or just a Zeta-Jones effect? An exploratory study." Psychiatry Research 228.3 (2015): 913-917.

27 - Arora, Vishal S., Martin McKee, and David Stuckler. "Google Trends: Opportunities and limitations in health and health policy research." Health Policy 123.3 (2019): 338-341.

28 - Vesel, Claudia, et al. "Effects of mood and aging on keystroke dynamics metadata and their diurnal patterns in a large open-science sample: A BiAffect iOS study." Journal of the American Medical Informatics Association 27.7 (2020): 1007-1018.

29 - Fancourt, Daisy, Andrew Steptoe, and Feifei Bu. "Trajectories of anxiety and depressive symptoms during enforced isolation due to COVID-19 in England: a longitudinal observational study." The Lancet Psychiatry 8.2 (2021): 141-149. 
30 - Brodersen, Kay H., et al. "Inferring causal impact using Bayesian structural time-series models." Annals of Applied Statistics 9.1 (2015): 247-274. 